\begin{tabular}{|ll|}
\hline Citation & $\begin{array}{l}\text { Bermúdez Pita, M., Ntegeka, V., Wolfs, V., Willems, P. } \\
\text { Development and comparison of two fast surrogate models for urban } \\
\text { pluvial flood simulations } \\
\text { Water Resources Management, doi:10.1007/s11269-018-1959-8 (2018) }\end{array}$ \\
\hline Archived version & $\begin{array}{l}\text { Author manuscript: the content is identical to the content of the published } \\
\text { paper, but without the final typesetting by the publisher }\end{array}$ \\
\hline Published version & $\underline{\text { https://link.springer.com/article/10.1007/s11269-018-1959-8 }}$ \\
\hline Journal homepage & $\underline{\text { https://link.springer.com/journal/11269 }}$ \\
\hline Author contact & \\
\hline IR & $\underline{\text { pattrick.willems @kuleuven.be }}$ \\
\hline
\end{tabular}

(article begins on next page) 


\title{
Development and comparison of two fast surrogate models for urban pluvial flood simulations
}

María Bermúdez ${ }^{1,2 *}$, Victor Ntegeka $^{1}$, Vincent Wolfs $^{1}$, Patrick Willems ${ }^{1}$

1 Hydraulics Section, Department of Civil Engineering, KU Leuven, Belgium

2 Water and Environmental Engineering Group, Universidade da Coruña, Spain

*corresponding author. Email: mbermudez@udc.es. Phone: +34 981167000, Ext. 1427. ORCID: 0000-0003-3189-4791

\begin{abstract}
Detailed full hydrodynamic 1D-2D dual drainage models are a well-established approach to simulate urban pluvial floods. However, despite modelling advances and increasing computational power, this approach remains unsuitable for many real time applications. We propose and test two computationally efficient surrogate models. The first approach links a detailed 1D sewer model to a GIS-based overland flood network. For the second approach, we developed a conceptual sewer and flood model using datadriven and physically based structures, and coupled the model to pre-simulated flood maps. The city of Ghent (Belgium) is used as a test case. Both surrogate models can provide comparable results to the original model in terms of peak surface flood volumes and maximum flood extent and depth maps, with a significant reduction in computing time.
\end{abstract}

Keywords: computational hydraulics; surrogate modelling; pluvial flooding; urban drainage model; urban flooding

\section{Acknowledgments}

The authors would like to thank the Spanish Regional Government of Galicia (Postdoctoral grant ED481B 2014/156), Agentschap Innoveren en Ondernemen (Vlaio) for funding, Innovyze for the InfoWorks ICM license, and the water company Farys for the original 1D sewer model of the area. The research is conducted within the project PLURISK for the Belgian Science Policy Office. 


\section{Introduction}

Full hydrodynamic 1D-2D dual drainage models are a well-established approach to simulate urban pluvial flooding, which can provide a realistic description of flood conditions. They simulate the coupling between the sewer network, represented by a detailed 1D model, and the surface flow, computed using a 2D hydrodynamic model. This approach is now available in commercial software packages such as SOBEK (Deltares 2017), XP-SWMM 2D (XP Solutions 2014), MIKE FLOOD (DHI 2012) or InfoWorks ICM (Innovyze 2015), and is commonly applied in urban flood studies (Schmitt et al. 2004; Jahanbazi and Egger 2014). However, such set up is generally too computationally intensive for real-time applications such as urban pluvial flood forecasting or for probabilistic approaches that require multiple simulations.

Therefore, there remains a need for developing computationally more efficient surrogates of these models. Two broad families of surrogate models can be considered: lower-fidelity models, which are simplified physically based models preserving the main body of processes modeled in the original system, and data-driven models which emulate the original model responses without analyzing the physical processes involved (Razavi et al. 2012). In the context of 1D-2D dual drainage models, lower fidelity approaches can be developed for the underground sewer model, the surface model, or both. However, given that the $2 \mathrm{D}$ surface flow model is generally more computationally expensive than the 1D sewer model, the approaches that rely solely on simplified representations of the sewer system do not necessarily result in a reduction of computation times. Therefore, most often lower fidelity approaches apply simplifications in both the sewer and the surface system.

The sewer system can be represented in the simplest approaches by subtracting a constant rate of rainfall (Environment Agency 2013; van Dijk et al. 2014) or by using a constant infiltration rate (Chen et al. 2009) that reflects the draining and storage capacity of the sewer. These approaches can be interesting if the underground drainage does not have much influence on the studied flood phenomenon 
or if network data are missing (Henonin et al. 2013). More complicated yet popular approach is skeletonisation (Leitão et al. 2010), in which secondary elements of the network model are removed or changed.

On the other hand, the urban surface system can be represented in the simplest approaches by virtual reservoirs, located on top of the manholes, which store the overflow volumes. This approach alone cannot be used for a realistic assessment of flood dynamics, but can give an estimation of potential overflow locations (Maksimovic and Prodanovic 2001). On the other hand, the urban surface can be represented by a network of open channels and ponds, forming a so-called 1D-1D dual drainage model when combined with a 1D sewer model (Maksimović et al. 2009). For complex topologies (e.g. multidirectional flow paths), a 2D treatment of the surface flow hydraulics can be necessary. Model complexity can then be reduced by solving simplified forms of the 2D shallow water equations (Neal et al. 2012), applying grid coarsening methods in combination with a subgrid model (Schubert and Sanders 2012), or using nested grids (Bermúdez et al. 2017). However, these models may still not fully satisfy the needs of city emergency management due to model complexity, setup data requirements and computing times. Further simplifications have thus been proposed in recent years, such as urban inundation models based on geographic information systems (Zhang and Pan 2014; Jahanbazi and Egger 2014) or on a cellular automata approach (Liu and Pender 2013; Ghimire et al. 2013).

The physically-based models described above are being more and more complemented by data-driven models (Solomatine and Ostfeld 2008), which use machine learning methods to approximate the response of the original model. In the water resources field, artificial neural networks (ANN) are a popular approximation technique (Razavi et al. 2012). They have been widely and successfully applied for modelling rainfall-runoff processes, forecasting streamflow (Yaseen et al. 2015) and approximating rating curves (Wolfs and Willems 2014), but applications to urban hydraulics and hydrology are still scarce ( $\mathrm{Li}$ et al. 2010). ANN models have been applied to predict flooding at the nodes of a sewer network based on rainfall input (Duncan et al. 2011, 2013), or to emulate Combined Sewer Overflow 
(CSO) dynamics and water quality variables (Keupers and Willems 2015). (Wolfs and Willems 2017) recently developed a conceptual sewer modelling approach which divides the sewer system into interconnected cells and uses ANN models to estimate flows between them. All the above studies have reported significant speed gains over conventional hydrodynamic models with an acceptable loss of accuracy for most intended applications, which highlights the potential of this approach for predicting urban flooding in real-time. The generalization ability beyond the training data is nevertheless a concern when applying this type of models.

This study elaborates on the suitability of lower fidelity and data driven techniques for urban pluvial flood modelling. We propose and test two novel surrogate models of a computationally demanding 1D-2D dual drainage model, for practical applications that require results in a very short time. The first model relies on lower fidelity simplifications to represent the surface system. The second model combines both physically-based and data driven modelling approaches to develop a conceptual sewer and flood model. The capabilities of these two models for pluvial flood inundation mapping are evaluated and compared.

\section{Methodology}

\subsection{Case study}

The city of Ghent in Belgium was used as a test case (Figure 1a). The studied area covers $27.5 \mathrm{~km}^{2}$ of flat terrain and is highly urbanized, with a population equivalent of 43,626 . Four regions of the study area, with sizes around $0.3 \mathrm{~km}^{2}$, were defined to develop the surrogate models (Figure 1b). Model performance was evaluated considering 4 rainfall events: 2 historical events, occurred on 28 th July 2013 and 30th May 2016, and 2 events obtained with a stochastic rainfall generator (Muñoz et al. 2015). To set up surrogate model 2 (see $\$ 2.4$ ), a total of 120 storms based on the rainfall data of the meteorological station of the Royal Meteorological Institute of Belgium at Uccle were created and employed. This additional data enhances the generalization capabilities of the data-driven modelling 
approach, and can increase its accuracy. The 120 storms are based on different historical storms with large rainfall intensities (peak 10-min rainfall intensities above $15 \mathrm{~mm} / \mathrm{h}$ ) and/or cumulative volumes (independent events with the highest 6-hour cumulative rainfall). Out of the 120 storms, 15 were created by merging 2 separate storms, leading to events with two consecutive peaks. Such succession of peaks can have a major impact of urban flooding, as storages (in the sewer and on the surface) can already be filled after the first peak. Also, the rainfall intensities of most storms were artificially increased ( $+10 \%$ to $40 \%)$ to cause flooding, and thus create additional flood calibration data for surrogate model 2. Additionally, 8 synthetic storms (Willems 2013) with return periods from 2 to 100 years are used to derive pre-simulated flood maps.

\subsection{D-2D dual drainage model}

A detailed 1D-2D full hydrodynamic model of the sewer network and the surface of the city was implemented in InfoWorks ICM (Figure 1b). The model covers an area of $27.5 \mathrm{~km}^{2}$ and is comprised of 6025 conduits, 182 hydraulic structures and 5855 manholes. The resolution of the surface triangular mesh ranged from $3.75 \mathrm{~m}^{2}$ up to $50 \mathrm{~m}^{2}$ in the flood prone areas. The interaction between the 1D underground sewer conduits and the 2D surface was through the manholes, conceptualized as weirs. The double linear reservoir model (or Wallingford model) was used to route the flow from the surface to the manholes based on the input rainfall. Calibration was performed according to the guidelines of the Hydronaut procedure (Aquafin 2005), which is a methodology that is commonly used to configure hydrodynamic sewer models in Belgium. This high-fidelity physics-based model is assumed to be the best representation of the system behavior for the purposes of this work, as is commonly done when developing surrogate models (Kroll et al. 2017).

\subsection{Surrogate model 1}

Two different surrogate models of the above model were developed in this work (Figure 2). The first surrogate model consists of a $1 \mathrm{D}$ representation of the sewer network in which the flood volumes are 
stored in virtual reservoirs on top of the manholes. A conical flood storage volume was defined for each manhole, specifying a cross-sectional area versus height relation. Two alternative definitions were implemented and tested: a default definition based on the size of the contributing areas draining to each node and an arbitrary total height of $100 \mathrm{~m}$ (named surrogate model 1A), and an enhanced definition based on the floodable areas estimated from the surface topography (named surrogate model 1B).

The GIS mapping (Figure 2, Surrogate model 1) consists of a flood volume spreading algorithm that translates the maximum flood volume from surcharged manholes into a flood depth map. The method is based on an iterative algorithm that moves flood volumes over the terrain from grid cell to grid cell of the raster digital elevation model (DEM), and is similar to the procedure applied in (Shapiro and Westervelt 1992; Shook et al. 2013). The approach assumes that the drainage connectivity depends on the DEM. Thus, efforts must be made to minimize the potential connectivity inaccuracies such as areas with culverts or bridge crossings.

The flood volume spreading algorithm operates as follows. At each iteration, a fraction of the depth in a grid cell can be drained to the eight neighbouring grid cells. This means that the central cell is compared to each neighbouring cell separately without selecting a predominant flow direction. However, as some regions are rarely flooded, calculations can be restricted to those grid cells within flood prone regions. This flood zone can be defined based on historical flood maps or pre-simulated maps. For this study, a 100-year return period storm event was simulated in the 1D-2D dual drainage model and was used to identify flood zones. A buffer of $10 \mathrm{~m}$ was added to include more neighbouring cells. Within the flood zone, a height difference is calculated between the selected grid cell and its neighbouring grid cells. The height is calculated as the summation of the water depth and the ground elevation. If the neighbouring grid cell's height is greater than the selected grid cell's height, the selected grid cell receives a fraction of the height difference, otherwise the neighbouring grid cell receives it. The fraction used for transfer was taken as 10 percent. This fraction is estimated based on 
the study area characteristics and computation time. It is meant to aid the iterative procedure of spatially distributing volumes between cells. One could depend on some physical characteristics such as topography as a guide. Higher volume exchanges would be likely in areas with higher slopes, for instance. However, a lower fraction would mean less volume exchange which would require more iterations, and hence additional calculation time, to achieve the required flood depth map.

Since such calculations are computationally expensive, the distribution of the volumes can be implemented using parallel computing. To allow for this, grid cells are grouped to avoid overlapping calculations. A total of 9 group simulations can be run concurrently to allow for a faster calculation of the flood extent. Figure 3 shows a group of grid cells that meet the non-overlapping criteria. After each iteration, it is important to verify that the change in water depth to the previous iteration is within a tolerance limit. The threshold for convergence was set at $10 \mathrm{~mm}$ to allow for a realistic representation of the final water depth. This threshold was selected on the basis of the level of accuracy for flood extents and the calculation time. A lower convergence threshold would be more accurate for flood extents but would require higher calculation times. Figure 3 illustrates the flood spreading procedure. The initial water depth map prior to spreading was calculated by estimating the flood depth at the manhole locations. For manhole locations that are close to each other (within 2 meters), the volumes are combined and then spread on the grid cells close to the flooded grid cells (as shown in area 3 in Figure 3). Initial depths are usually high but after several iterations, the volume is spread over the terrain and realistic flood depths are achieved.

\subsection{Surrogate model 2}

The second surrogate model combines a conceptual lumped hydraulic sewer model and a simplified flood model, using both data-driven and physically based model structures. Given rainfall and nearby river level series, the model yields volumes and maps at different locations in the city. The surrogate model was calibrated using simulation results of the detailed full hydrodynamic 1D-2D InfoWorks 
ICM model. The same rainfall runoff routing was used as in surrogate model 1. If such detailed 1D2D model is unavailable, one could also calibrate this model to measurements. However, the level of model detail and its accuracy will depend on the availability and reliability of such measurements.

The underground system is emulated using the conceptual modelling approach described in (Wolfs and Willems 2017). First, the sewer network is divided into interconnected storage cells, each representing different parts of the sewer system. The conceptual model topology was chosen such that it minimizes flows between cells, which finally resulted in a model consisting of 13 storage cells (Figure 1c). Experiments also showed that lumping processes further and creating fewer cells could lead to inaccurate model predictions. Next, the flows between the storage cells were emulated using a variety of model structures, including neural networks, transfer functions and piecewise linear relationships (see (Wolfs and Willems 2017) for further information on the model structures and calibration algorithms). For each flow path, the most appropriate model structure was chosen based on the dynamics of the system. The model outcomes are volumes in each storage cell, and discharges at several locations.

The flood model estimates surface flood volumes, aggregated in the pre-defined regions of the study area (Figure 1b), using a serial connection of two artificial neural networks (ANNs). The first is a neural classification network that can identify when flooding from the underground system emerges. This network holds 10 hidden neurons arranged in one layer, and was trained using the scaled conjugate gradient approach minimizing cross-entropy. Next, an ensemble of five feedforward neural networks quantifies the magnitude of the flood. Using such ensemble reduces the risk of overfitting and increases the generalization capabilities. These ANNs have between 15 and 25 hidden neurons configured in one hidden layer. The flood model distinguishes flood volumes which are connected directly to the sewer system and thus can drain quickly, and flood volumes stored in local depressions which can only be emptied slowly via infiltration and evaporation. Only the former volumes are predicted directly via the ANN ensemble, which can be used to deduce the other volumes via GIS 
processing. Both ANNs use the same three inputs, which are the rainfall runoff volumes aggregated over 10 and 30 minutes windows, and the volume in the underground system of the closest storage cell.

In the final step of the modelling cascade, the simulated flood volumes are translated into flood depth maps (Figure 2). Pre-simulated scenarios, corresponding to synthetic storms with return periods from 2 to 100 years, are used to predict the spatial distribution of flooding within each region. The method uses the flood volume simulated by the surrogate model as input for scenario selection.

\section{Results}

The performance of the surrogate models is compared to that of the original 1D-2D model, first in terms of computational cost. The simulation times of the three models differ significantly. The 1D-2D dual drainage model takes approximately $408 \mathrm{CPU}$ minutes to simulate a 6-hour event on an i7 processor at $3.40 \mathrm{GHz}$ and $16 \mathrm{~GB}$ RAM. Surrogate model 1 takes around $91 \mathrm{CPU}$ minutes to estimate surface flood volumes in such an event, the flood mapping step adding of the order of 60 minutes. The surrogate model 2 requires less than a second overall. This vast speed gain would enable numerous applications that require a large number of real time simulations, provided that the loss of accuracy is acceptable. This aspect is evaluated in the following.

The results of the surrogate models are compared with those obtained with the 1D-2D dual drainage model. Given the limited field data available, the 1D-2D dual drainage model is considered to provide the more accurate representation of the system behavior. The comparison is performed for each predefined region in terms of: (1) evolution of total flood volume in the surface, (2) flood inundation and depth maps corresponding to the maximum surface flood volume.

\subsection{Flood volume simulation}


The results show that the first surrogate model is sensitive to the definition of the virtual storage reservoirs in the sewer model (Figure 4). With the default definition (model 1A), the surface flood volumes are significantly underestimated, being the peak flood volumes between $30 \%$ and $67 \%$ lower than those obtained with the 1D-2D dual drainage model (Table 1). When the definition of the virtual storage is based on the floodable areas estimated from the surface topography (model 1B), the maximum flood volumes are better estimated. The maximum underestimation of peak flood volumes is $27 \%$ (Table 1$)$.

Surrogate model 1 can predict the start of the flood event, but is unable to emulate the behavior of the surface flow, as shown by the low values of the Nash-Sutcliffe efficiencies in Table 1. The surcharged volumes return to the sewer system more quickly than with the $1 \mathrm{D}-2 \mathrm{D}$ dual drainage model, resulting in lower flood durations (as in R4 in Figure 4) and underestimation of peak flood volumes in multiple peak events (as in R3 in Figure 4). The straightforward explanation is that this model does not replicate storage in local depressions that can only slowly infiltrate or evaporate, and thus cannot be used to predict the evolution of the volumes at the surface.

Surrogate model 2 emulates the results of the 1D-2D dual drainage model accurately, in terms of flood volumes, in regions 1,2 and 4. The differences in peak flood volumes with respect to the simulations of the 1D-2D model are below $20 \%$ in these regions. The model can provide accurate estimations not only of the maximum volume, but also of its evolution during the flood event, as reflected in the higher NSE values (above 0.79 in these regions, Table 1). In region 3, although the model can correctly identify the occurrence of a flood event, the flood volume simulations are poor. The surrogate model succeeds in predicting the moment of inundation correctly, but overestimates the maximum flood volume for events May 2016, July 2013 and event B. Performance can be increased by using more advanced neural networks (e.g. a higher number of neurons) and including more training data. In any case, the flood duration is emulated more accurately by the surrogate model 2 than with the surrogate 
model 1. It is clear that the simulation of the receding flood volume is more realistic with the surrogate model 2.

\subsection{Flood inundation mapping}

In order to compare the predictions of inundation extent obtained with the surrogate models and the 1D-2D dual drainage model, we employ the precision (p), the recall ( $\mathrm{r}$ ) and the F1 score as performance measures, defined as follows:

$\mathrm{p}=\frac{\mathrm{A}}{\mathrm{B}}$

$\mathrm{r}=\frac{\mathrm{A}}{\mathrm{C}}$

$\mathrm{F} 1=2 \frac{\mathrm{p} \cdot \mathrm{r}}{\mathrm{p}+\mathrm{r}}$

where A is the area correctly predicted as flooded by the surrogate model, B is the total area predicted as flooded by the surrogate model and $\mathrm{C}$ is total area predicted as flooded by the 1D-2D dual drainage model. A wet-dry threshold of $0.01 \mathrm{~m}$ is considered to delineate the flooded areas. A high precision $p$ means that most of the area that the surrogate model predicts as flooded is also predicted as such by the 1D-2D dual drainage model. A high recall $r$ means that most of the flooded area predicted by the 1D-2D-dual drainage model is well captured by the surrogate model. In the context of early warning flood forecasting systems, a high recall ensures that the authorities are alerted to take action in most of the actual flooded areas, whereas a high precision avoids unnecessary actions and preparations in non-flooded areas. F1 is the harmonic mean of precision and recall, and is equal to 1 when the flooded areas predicted by the surrogate and the 1D-2D dual drainage model coincide exactly.

In order to evaluate the quality of water depth simulations of the surrogate models, the mean absolute error (MAE) of the flood depth simulations was computed. With this metric, the flood depth simulations of the surrogate models were compared with the results of the original $1 \mathrm{D}-2 \mathrm{D}$ dual drainage model. The lower the MAE value, the better the agreement between the simulations of the 
two models. All the above metrics are commonly used for evaluating flood inundation models (Aronica et al. 2002; Pappenberger et al. 2007; Liu and Pender 2013; Stephens et al. 2014).

The surrogate models developed in this work use the peak flood volume as input for flood mapping (Figure 2 and Figure 5). Given the poor estimations of peak volumes obtained with model 1A, only model $1 \mathrm{~B}$ and model 2 are compared at this stage. Table 2 shows the performance metrics obtained with both models. The surrogate models' simulations show a good overall agreement with the maps calculated with the 1D-2D dual drainage model. It should be noted that for certain events, surface flood volumes can be very low in some regions, and hence the derivation of a flood map is irrelevant (these cases are marked with an asterisk in table 2). For event A and region 4, the 1D-2D model predicts no flooding for that location, and both surrogate models correctly produce a zero flood depth map. In the remaining cases, surrogate model 2 shows a slightly better performance than surrogate model 1, due to the underestimation of peak flood volumes in the latter. Most of the area that the surrogate model 1 predicts as flooded is also predicted as such by the 1D-2D dual drainage model, but the 1D-2D dual drainage model is mapping a larger flood extent (as in R1 in Figure 5). This results in higher precision than recall (Table 2). Surrogate model 2 presents high precision and recall, with F1 scores above 0.81 and MAE of around $0.02 \mathrm{~m}$. The use of pre-simulated maps based on surface flood volumes can thus provide a reasonable characterization of the flood depth field at the street level.

\section{Discussion}

In the context of urban flood applications, the choice of a modeling strategy is mainly influenced by data availability and flood context (flood type, aimed application, etc.), and involves balancing accuracy, computation time, data needs and communication possibilities (Henonin et al. 2013; van Dijk et al. 2014). Therefore, the proposed modelling approaches are compared on these topics.

Both models can identify the occurrence of a flood and predict the moment of inundation correctly as evidenced by Table 1 and Figure 5. Surrogate model 1 is not well suited to simulate flood evolution 
and durations accurately, whereas surrogate model 2 can reproduce flood dynamics more precisely (see Figure 5). Regarding the spatial flood prediction, surrogate model 1 can identify which manholes are surcharged under the existing flow conditions. On the contrary, surrogate model 2 predicts the surface flood volumes lumped in pre-defined regions, so it is not possible to identify the precise overflow locations.

The flood maps predicted by the 1D-2D dual drainage model and the surrogate models compare well in terms of flood extent and flood depth. Both surrogate models use the peak flood volume as input for flood mapping. The flood maps thus reflect the errors in the estimation of peak flood volumes. In surrogate model 1 , the parameterization of the virtual reservoirs for each manhole has a significant impact on the simulated flood volumes, and the default parameterization underestimates flood volumes significantly at all locations. However, reasonably accurate flood maps can be produced with both mapping approaches (i.e., the flood volume spreading algorithm in surrogate model 1 and the presimulated maps in surrogate model 2) provided that good estimates of peak flood volumes are used. The mapping approaches proposed in this paper have a very simple setup and require few modelling choices. Almost all of the model configuration steps in both proposed approaches can be fully automated (if a detailed 1D/2D model is available), including the calibration of the flood module in surrogate model 2. The lack of required modelling choices in the proposed approaches constitutes an advantage over other methods such as the 1D representation of the urban surface, which requires predefining a network of flow paths and ponds. The latter is not straightforward and is subject to a high degree of uncertainty in very flat areas like the one studied here. The use of pre-simulated scenarios is more efficient than the GIS mapping procedure in terms of computational time, given that flood maps are instantly retrieved form a database. This approach can thus be particularly interesting for practical use in large urban areas.

However, the flood mapping approaches implemented in this study are not without limitations. The flood spreading approach assumes that flood volumes are distributed over the terrain without explicit 
consideration of physical processes. Hence, flood velocity and flood volume losses which may occur as flood volume spreads over the surface are not included. Nevertheless, the simplified flood spreading approach allows for a diagnostic assessment of flood prone areas as well as identifying flow path connectivity. For cases where flood hazard information is required in a relatively short time, such as emergency management, simplified flood volume spreading is of critical importance. It is important to note, however, that the accuracy of the results critically depends on the resolution and quality of the DEM. A better land surface representation helps to capture relevant features which minimizes anomalies in flood extents.

The speed gains obtained, which are simply the ratio between the computational time of the $1 \mathrm{D}-2 \mathrm{D}$ dual drainage model and the surrogate models, are in the order of 5 and $10^{4}$ for models 1 and 2, respectively. This speed gains, in particular with surrogate model 2, would enable numerous applications that require results in a very short time (e.g., real-time applications) or a large number of model simulations (e.g., optimization problems or uncertainty assessments).

Finally, the surrogate models presented here are comprised of several components or modules, as shown in Figure 2. As such, they can be interchanged or combined to define new modelling approaches. For example, the 1D representation of the sewer network with virtual reservoirs (as in surrogate model 1) can be coupled with pre-simulated flood maps (as in surrogate model 2). Similarly, components which are deemed unnecessary for a particular application can be removed. An example of the latter could be the definition of an urban flood warning system based only on the 1D the sewer network model with virtual reservoirs (as in surrogate model 1), without a flood mapping component.

\section{Conclusions}

Two surrogate modelling approaches of a highly detailed 1D-2D dual drainage model were developed and compared to simulate pluvial flooding on an urban area in Belgium. Surrogate model 1 is based on a 1D sewer network model, in which flood volumes are stored in virtual reservoirs on top of the 
manholes. Flood mapping is performed by means of a GIS volume spreading algorithm. Surrogate model 2 combines a conceptual lumped hydraulic sewer model with a simplified flood model, using both data-driven and physically based structures. Flood mapping is based on pre-simulated scenarios.

The first surrogate model can identify overflow locations and give an estimation of the maximum flood volume, provided that the parameterization of the virtual reservoirs reflects the urban surface topography. These data can then be used to derive a reasonably accurate maximum flood depth map based on the surface topography. The second surrogate model can emulate the evolution of the flood volumes accurately and can thus be used to predict the dynamics of the flood event. Pre-simulated maps show a good agreement with the flood maps predicted by the 1D-2D dual drainage model, in terms of flood extent and depth. Both surrogate models require shorter calculation times, surrogate model 2 showing clearly superior performance in this aspect, with speed gains above $10^{4}$. This vast speed gain and the reduced loss of accuracy demonstrate the great potential of the developed surrogate modelling approach for real-time use.

\section{Conflict of Interest - None}

\section{References}

Aquafin (2005) Hydronaut procedure, versie 6.0 (internal modelling guidelines, in Dutch)

Aronica G, Bates PD, Horritt MS (2002) Assessing the uncertainty in distributed model predictions using observed binary pattern information within GLUE. Hydrol Process 16:2001-2016 . doi: 10.1002/hyp.398

Bermúdez M, Neal JC, Bates PD, et al (2017) Quantifying local rainfall dynamics and uncertain boundary conditions into a nested regional-local flood modeling system. Water Resour Res 53:2770-2785 . doi: 10.1002/2016WR019903

Chen AS, Djordjevic S, Fowler HJ, et al (2009) Pluvial flood modelling of the South East London Resilience Zone in the Community Resilience to Extreme Weather (CREW) Project. In: Flood 
and Coastal Risk Management Conference. Telford, UK.

Deltares (2017) SOBEK. Hydrodynamics, Rainfall Runoff and Real Time Control. User Manual SOBEK 2.15.

DHI (2012) MIKE FLOOD User Manual

Duncan A, Chen AS, Keedwell E, et al (2013) RAPIDS: Early Warning System for Urban Flooding and Water Quality Hazards. In: Machine Learning in Water Systems symposium: part of AISB Annual Convention 2013, University of Exeter, UK, 3-5 April 2013

Duncan AP, Chen AS, Keedwell EC, et al (2011) Urban flood prediction in real-time from weather radar and rainfall data using artificial neural networks. In: Weather Radar and Hydrology International Symposium. IAHS Red Book Series no. 351

Environment Agency (2013) What is the updated Flood Map for Surface Water? Bristol, UK Ghimire B, Chen AS, Guidolin M, et al (2013) Formulation of a fast 2D urban pluvial flood model using a cellular automata approach. J Hydroinformatics 15:676 . doi: 10.2166/hydro.2012.245

Henonin J, Russo B, Mark O, Gourbesville P (2013) Real-time urban flood forecasting and modelling - a state of the art. J Hydroinformatics 15:717-736

Innovyze (2015) InfoWorks ICM Help v5.5

Jahanbazi M, Egger U (2014) Application and comparison of two different dual drainage models to assess urban flooding. Urban Water J 11:584-595 . doi: 10.1080/1573062X.2013.871041

Keupers I, Willems P (2015) CSO water quality generator based on calibration to WWTP influent data. In: Proceedings of the 10th International Conference on Urban Drainage Modelling. Québec, pp 97-104

Kroll S, Wambecq T, Weemaes M, et al (2017) Semi-automated buildup and calibration of conceptual sewer models. Environ Model Softw 93:344-355 . doi:

10.1016/j.envsoft.2017.02.030

Leitão JP, Simões NE, Maksimović Č, et al (2010) Real-time forecasting urban drainage models: full 
or simplified networks? Water Sci Technol 62:2106-2114 . doi: 10.2166/wst.2010.382

Li X, Zhou F, Lodewyk S (2010) Applications of Artificial Neural Networks in Urban Water System. In: Watershed Management 2010. American Society of Civil Engineers, Reston, VA, pp 508-519

Liu Y, Pender G (2013) Carlisle 2005 urban flood event simulation using cellular automata-based rapid flood spreading model. Soft Comput 17:29-37 . doi: 10.1007/s00500-012-0898-1

Maksimovic C, Prodanovic D (2001) Modelling of Urban Flooding—Breakthrough or Recycling of Outdated Concepts. In: Urban Drainage Modeling. American Society of Civil Engineers, Reston, VA, pp 1-9

Maksimović Č, Prodanović D, Boonya-Aroonnet S, et al (2009) Overland flow and pathway analysis for modelling of urban pluvial flooding. J Hydraul Res 47:512-523 . doi:

$10.1080 / 00221686.2009 .9522027$

Muñoz C, Wang LP, Willems P (2015) Towards a high resolution stochastic rainfall generator for urban applications. In: Molnar P, Peleg. N (eds) Proceedings of the 10th International Workshop on Precipitation in Urban Areas

Neal J, Villanueva I, Wright N, et al (2012) How much physical complexity is needed to model flood inundation? Hydrol Process 26:2264-2282 . doi: 10.1002/hyp.8339

Pappenberger F, Frodsham K, Beven K, et al (2007) Fuzzy set approach to calibrating distributed flood inundation models using remote sensing observations. Hydrol Earth Syst Sci 11:739-752 . doi: 10.5194/hess-11-739-2007

Razavi S, Tolson BA, Burn DH (2012) Review of surrogate modeling in water resources. Water Resour Res 48:W07401 . doi: 10.1029/2011WR011527

Schmitt TG, Thomas M, Ettrich N (2004) Analysis and modeling of flooding in urban drainage systems. J Hydrol 299:300-311 . doi: 10.1016/j.jhydrol.2004.08.012

Schubert JE, Sanders BF (2012) Building treatments for urban flood inundation models and 
implications for predictive skill and modeling efficiency. Adv Water Resour 41:49-64 . doi: 10.1016/j.advwatres.2012.02.012

Shapiro M, Westervelt J (1992) R.MAPCALC An Algebra for GIS and Image Processing. U.S. Army Construction Engineering Research Laboratory. Champaign, IL

Shook K, Pomeroy JW, Spence C, Boychuk L (2013) Storage dynamics simulations in prairie wetland hydrology models: evaluation and parameterization. Hydrol Process 27:1875-1889 . doi: 10.1002/hyp.9867

Solomatine DP, Ostfeld A (2008) Data-driven modelling: some past experiences and new approaches. J Hydroinformatics 10:3-22 . doi: 10.2166/hydro.2008.015

Stephens E, Schumann G, Bates P (2014) Problems with binary pattern measures for flood model evaluation. Hydrol Process 28:4928-4937 . doi: 10.1002/hyp.9979

van Dijk E, van der Meulen J, Kluck J, Straatman JHM (2014) Comparing modelling techniques for analysing urban pluvial flooding. Water Sci Technol 69:305-311 . doi: 10.2166/wst.2013.699

Willems P (2013) Revision of urban drainage design rules after assessment of climate change impacts on precipitation extremes at Uccle, Belgium. J Hydrol 496:166-177 . doi:

10.1016/J.JHYDROL.2013.05.037

Wolfs V, Willems P (2014) Development of discharge-stage curves affected by hysteresis using time varying models, model trees and neural networks. Environ Model Softw 55:107-119 . doi: 10.1016/j.envsoft.2014.01.021

Wolfs V, Willems P (2017) Modular Conceptual Modelling Approach and Software for Sewer Hydraulic Computations. Water Resour Manag 31:283-298 . doi: 10.1007/s11269-016-1524-2 XP Solutions (2014) XP SWMM. Stormwater \& Wastewater Management Model. Getting started manual.

Yaseen ZM, El-shafie A, Jaafar O, et al (2015) Artificial intelligence based models for stream-flow forecasting: 2000-2015. J Hydrol 530:829-844 
Zhang S, Pan B (2014) An urban storm-inundation simulation method based on GIS. J Hydrol 517:260-268 . doi: 10.1016/j.jhydrol.2014.05.044 


\section{TABLES}

Table 1. Nash-Sutcliffe efficiencies (NSE) indicating the fit of the surface volumes calculated with the surrogate models to the results of the 1D-2D dual drainage model. NSE efficiency is evaluated over a period of between 90 and 180 minutes, depending on the event and region. Differences in peak surface volumes (4vol) in absolute and relative terms. Surface volumes are spatially aggregated in regions (R1 to $R 4$ as indicated in Figure $1 b)$.

\begin{tabular}{|c|c|c|c|c|c|c|c|c|c|c|c|c|c|}
\hline \multirow[b]{2}{*}{ Event } & \multirow[b]{2}{*}{ Metrics } & \multicolumn{4}{|c|}{ Model 1A } & \multicolumn{4}{|c|}{ Model 1B } & \multicolumn{4}{|c|}{ Model 2} \\
\hline & & R1 & R2 & R3 & R4 & R1 & R2 & R3 & R4 & R1 & R2 & R3 & R4 \\
\hline \multirow{3}{*}{ May 2016} & NSE & -0.52 & 0.67 & -2.47 & -1.33 & 0.66 & 0.80 & -2.06 & -0.63 & 0.98 & 0.97 & -1.17 & 0.80 \\
\hline & $\Delta \mathrm{vol}(\mathrm{m} 3)$ & -539 & -597 & -57 & -46 & -239 & -32 & -31 & -15 & 29 & -157 & 136 & 12 \\
\hline & $\Delta \operatorname{vol}(\%)$ & -60 & -38 & -30 & -65 & -27 & -2 & -16 & -21 & 3 & -10 & 71 & 17 \\
\hline \multirow{3}{*}{ July 2013} & NSE & -0.16 & 0.54 & -0.57 & -0.25 & 0.61 & 0.97 & -0.37 & 0.42 & 0.96 & 0.94 & 0.61 & 0.94 \\
\hline & $\Delta \operatorname{vol}(\mathrm{m} 3)$ & -198 & -234 & -45 & -80 & -80 & -82 & -23 & -26 & -39 & -87 & 39 & -1 \\
\hline & $\Delta \mathrm{vol}(\%)$ & -52 & -40 & -43 & -67 & -21 & -14 & -23 & -22 & -10 & -15 & 37 & -1 \\
\hline \multirow{3}{*}{ Event A } & NSE & -0.13 & 0.52 & 0.32 & - & 0.42 & 0.96 & 0.68 & - & 0.89 & 0.94 & 0.88 & - \\
\hline & $\Delta \mathrm{vol}(\mathrm{m} 3)$ & -197 & -576 & -627 & 0 & -107 & -175 & 72 & 0 & -82 & -116 & -91 & 0 \\
\hline & $\Delta \operatorname{vol}(\%)$ & -48 & -40 & -28 & - & -26 & -12 & 3 & - & -20 & -8 & -4 & - \\
\hline \multirow{3}{*}{ Event B } & NSE & -0.26 & 0.29 & -2.72 & 0.10 & 0.55 & 0.92 & -2.66 & 0.30 & 0.94 & 0.90 & -0.32 & 0.79 \\
\hline & $\Delta \operatorname{vol}(\mathrm{m} 3)$ & -406 & -264 & -2 & -135 & -152 & -70 & 0 & 33 & -140 & -17 & 14 & -136 \\
\hline & $\Delta \mathrm{vol}(\%)$ & -43 & -35 & -18 & -19 & -16 & -9 & -3 & 5 & -15 & -2 & 104 & -19 \\
\hline
\end{tabular}


Table 2. Performance of the surrogate models based on the simulated flood inundation and depth maps: precision (p), recall ( $r), F 1$ score and mean absolute depth error (MAE). Results corresponding to maps with surface flood volumes $<200 \mathrm{~m}^{3}$ are marked with an asterisk (*).

\begin{tabular}{|c|c|c|c|c|c|c|c|c|c|}
\hline \multirow{2}{*}{ Event } & \multirow[b]{2}{*}{ Metrics } & \multicolumn{4}{|c|}{ Model 1B } & \multicolumn{4}{|c|}{ Model 2} \\
\hline & & R1 & R2 & R3 & R4 & R1 & R2 & R3 & R4 \\
\hline \multirow{4}{*}{ May 2016} & $\mathrm{p}$ & 0.75 & 0.82 & $0.38^{*}$ & $0.57^{*}$ & 0.93 & 0.78 & $0.47^{*}$ & $0.93^{*}$ \\
\hline & $\mathrm{r}$ & 0.47 & 0.78 & $0.29 *$ & $0.45^{*}$ & 0.92 & 0.90 & $0.67^{*}$ & $0.87 *$ \\
\hline & F1 & 0.58 & 0.80 & $0.33^{*}$ & $0.50^{*}$ & 0.92 & 0.84 & $0.55^{*}$ & $0.90^{*}$ \\
\hline & MAE (m) & 0.06 & 0.04 & $0.04 *$ & $0.03 *$ & 0.02 & 0.03 & $0.03 *$ & $0.01^{*}$ \\
\hline \multirow{4}{*}{ July 2013} & $\mathrm{p}$ & 0.73 & 0.80 & $0.57^{*}$ & $0.71^{*}$ & 0.91 & 0.91 & $0.57^{*}$ & $0.96^{*}$ \\
\hline & $\mathrm{r}$ & 0.52 & 0.59 & $0.41^{*}$ & $0.53^{*}$ & 0.89 & 0.91 & $0.40^{*}$ & $0.58^{*}$ \\
\hline & F1 & 0.61 & 0.68 & $0.48^{*}$ & $0.60^{*}$ & 0.90 & 0.91 & $0.47^{*}$ & $0.72 *$ \\
\hline & MAE (m) & 0.05 & 0.05 & $0.03^{*}$ & $0.04 *$ & 0.02 & 0.01 & $0.02 *$ & $0.02 *$ \\
\hline \multirow{4}{*}{ Event A } & $\mathrm{p}$ & 0.72 & 0.77 & 0.78 & - & 0.93 & 0.94 & 0.83 & - \\
\hline & $\mathrm{r}$ & 0.54 & 0.66 & 0.63 & - & 0.87 & 0.86 & 0.67 & - \\
\hline & F1 & 0.62 & 0.71 & 0.70 & - & 0.90 & 0.90 & 0.74 & - \\
\hline & $\operatorname{MAE}(\mathrm{m})$ & 0.05 & 0.04 & 0.06 & - & 0.02 & 0.02 & 0.04 & - \\
\hline \multirow{4}{*}{ Event B } & $\mathrm{p}$ & 0.77 & 0.70 & $0.45^{*}$ & 0.77 & 0.92 & 0.94 & $0.19^{*}$ & 0.97 \\
\hline & $\mathrm{r}$ & 0.60 & 0.61 & $0.36^{*}$ & 0.59 & 0.83 & 0.71 & $0.99 *$ & 0.71 \\
\hline & F1 & 0.67 & 0.65 & $0.40^{*}$ & 0.67 & 0.87 & 0.81 & $0.32 *$ & 0.82 \\
\hline & $\operatorname{MAE}(\mathrm{m})$ & 0.06 & 0.04 & $0.03 *$ & 0.04 & 0.02 & 0.02 & $0.02 *$ & 0.02 \\
\hline
\end{tabular}




\section{FIGURES}
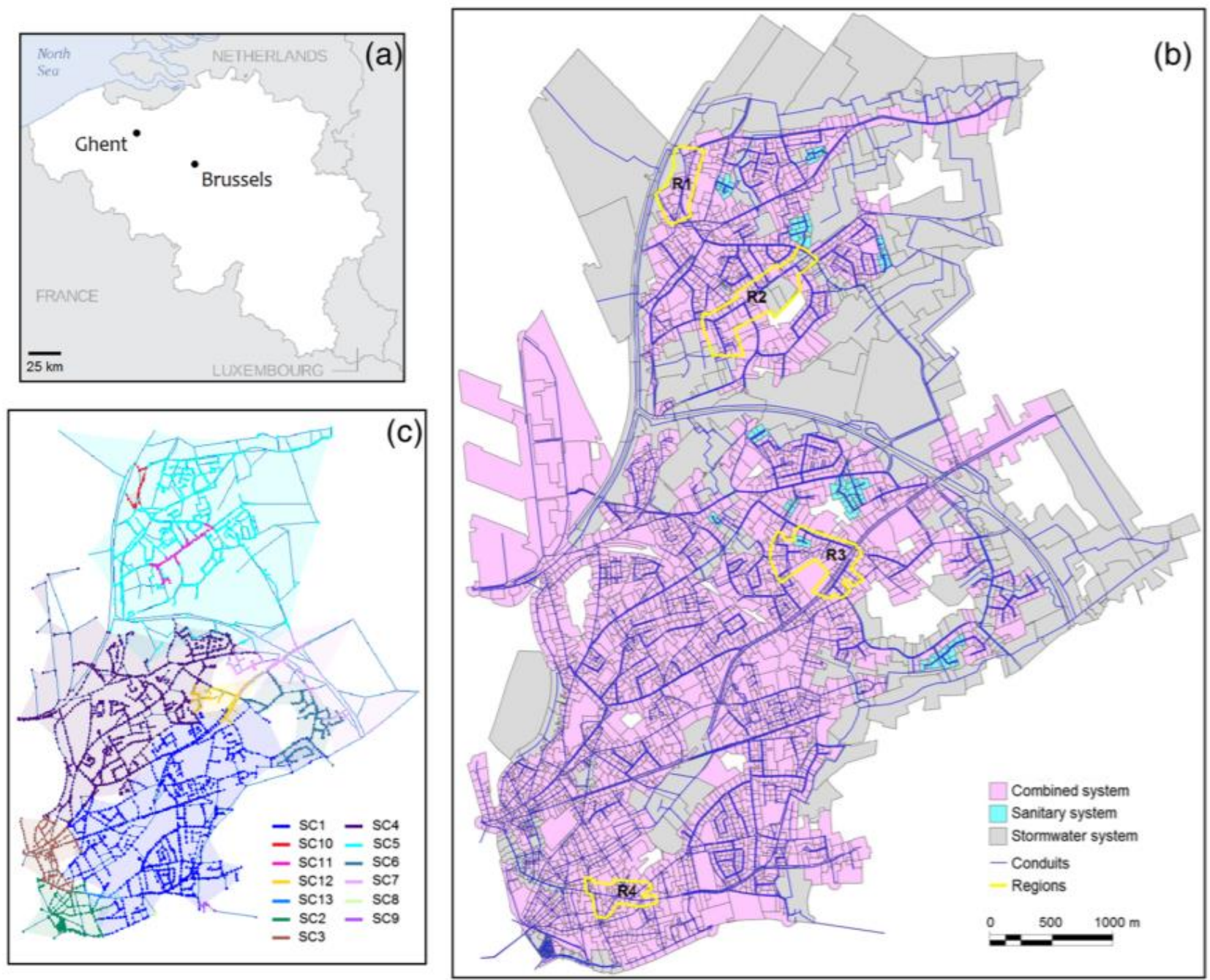

Fig. 1 (a) Location of Ghent in Belgium (adapted from UN-OCHA map); (b) Definition of the sewer network in the 1D-2D original model. The four regions analyzed are marked in yellow; (c) Division of the entire area in storage cells (SCs) for the underground sewer hydraulic model in surrogate model 2. 


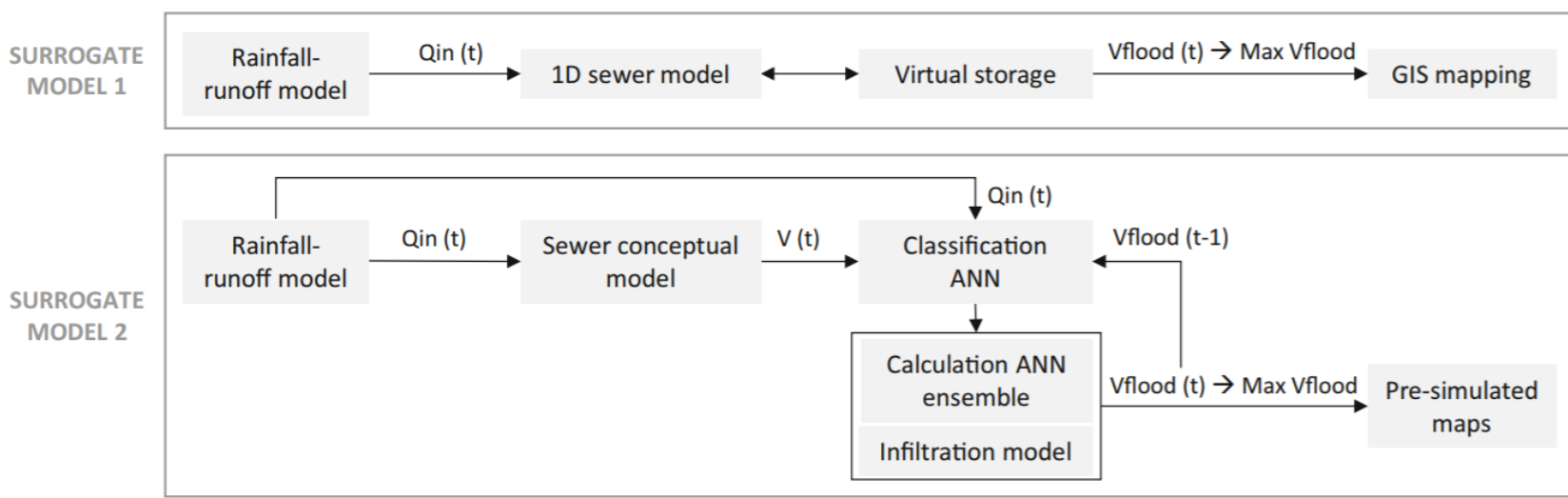

Fig. 2 Schematic overview of the different sub-models that comprise the two surrogate models and their main input and output data: the runoff inflows Qin, the volumes in the sewer system $\mathrm{V}$ and the flooded volumes Vflood
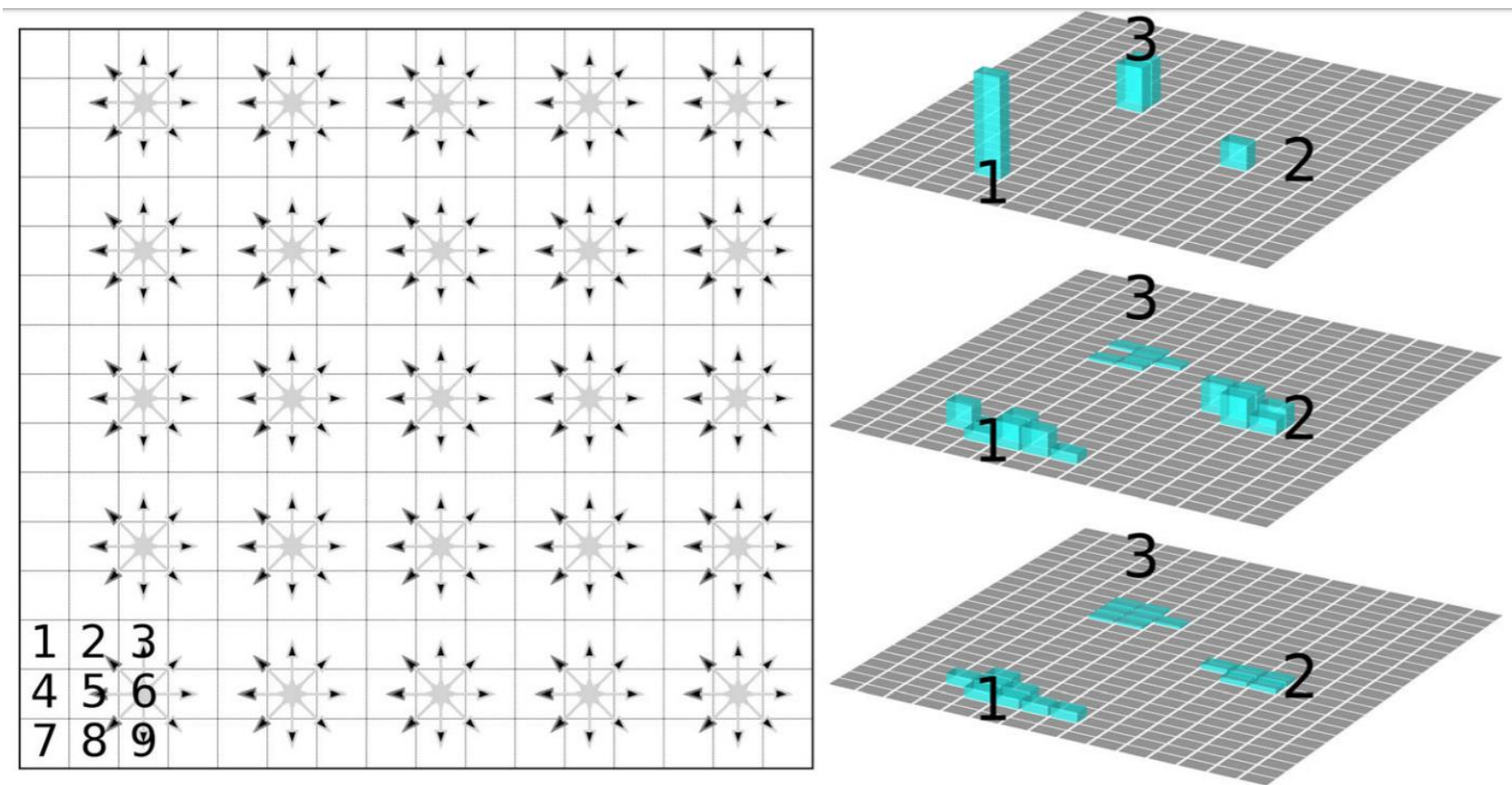

Fig. 3 Flood volume spreading for selected non overlapping grid cells of group 6 (centre cell in grey), and neighbouring cells (shown with black arrows) (left panel). Flood volume spreading at three time moments (right panel): initial flood depth (top panel), intermediate flood depth (middle panel) and final depth (bottom panel). For simplicity, topography is shown as a flat surface, although in practice the grey cells have different elevations based on the DEM 

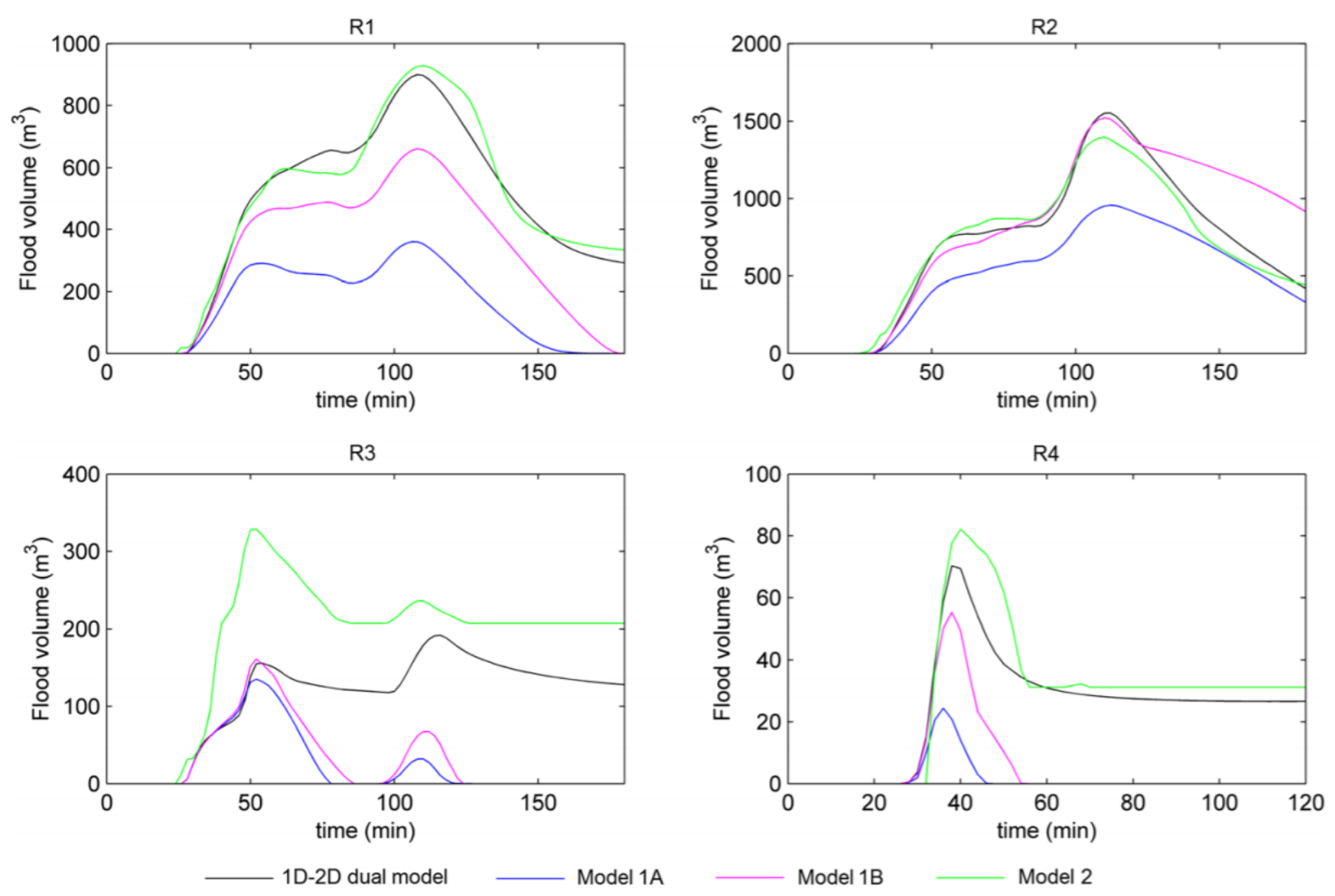

Fig. 4 Surface flood volumes simulated by the 1D-2D dual drainage model and the surrogate models for the May-2016 rainfall event. Surface volumes are spatially aggregated in regions (R1 to R4 as indicated in Figure 1b). 


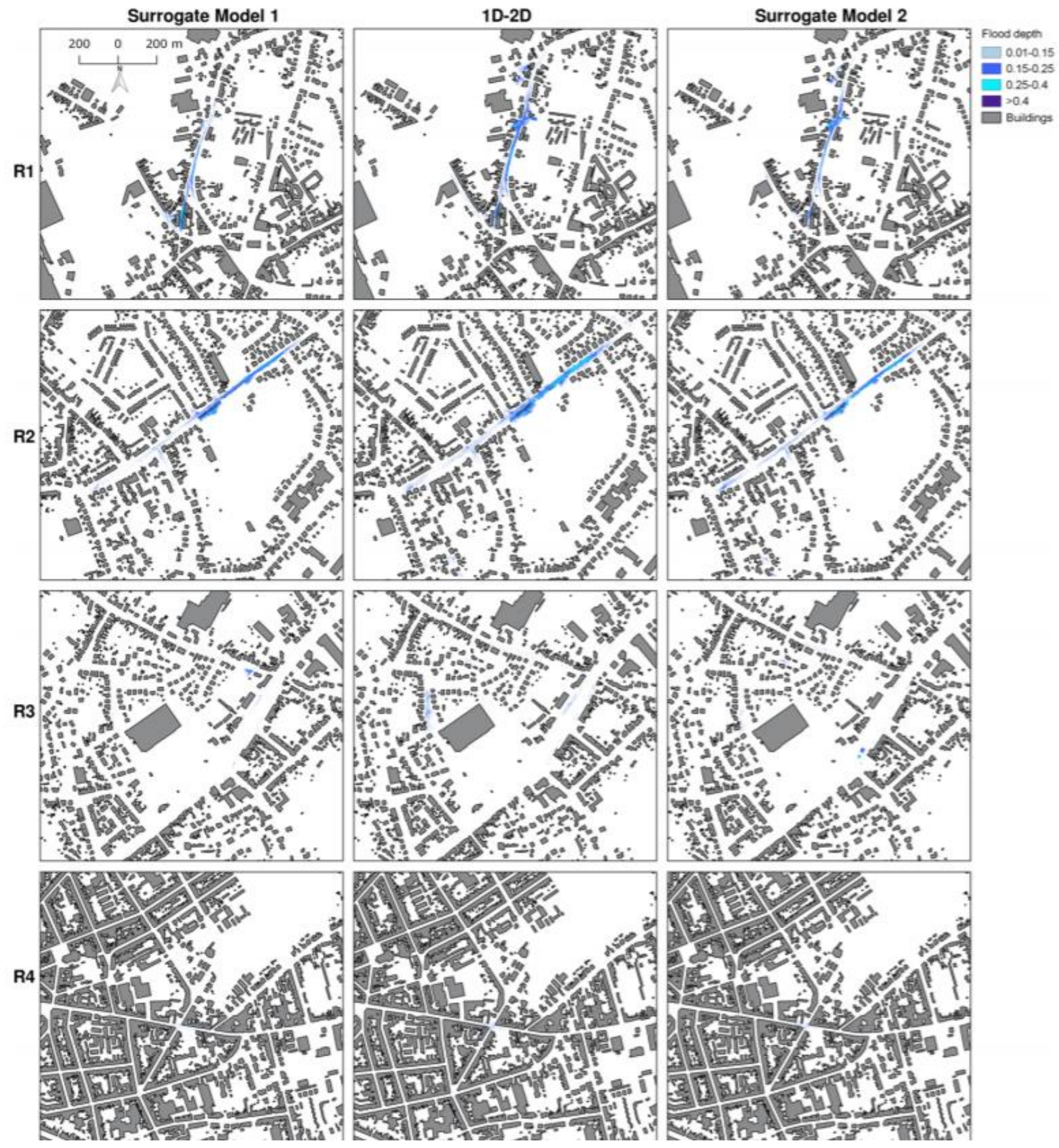

Fig. 5 Flood depth maps obtained with the surrogate model 1 (left), the 1D-2D dual drainage model (middle) and the surrogate model 2 (right) for May-2016 flood event. Regions R1 to R4 (up to down). As noted in Table 2, surface flood volumes for this event are below $200 \mathrm{~m}^{3}$ in regions R3 and R4. 